\section{AN IMPROVED ADJUSTABLE ELECTRIC LIGHT STAND FOR OPERATING ROOMS.} GILBERT ROYCE, B.A., M.B.

Formerly House Surgeon to the Eye and Ear Departments of the New York Eye and Ear Infirmary. TORONTO.

A feature of this standard is its universal adjustability. The whole stand can be rotated on its base. The height of the lamp can be regulated by the inner tube of the upright. The extension arm can be shortened or lengthened, tilted up or down or shortened and used vertically.

The standard consists of a heavy tripod base mounted on rubber-tired castors. Attached to this is an upright piece consisting of two tubes, one telescoping in the other, the movement of the inner tube being controlled

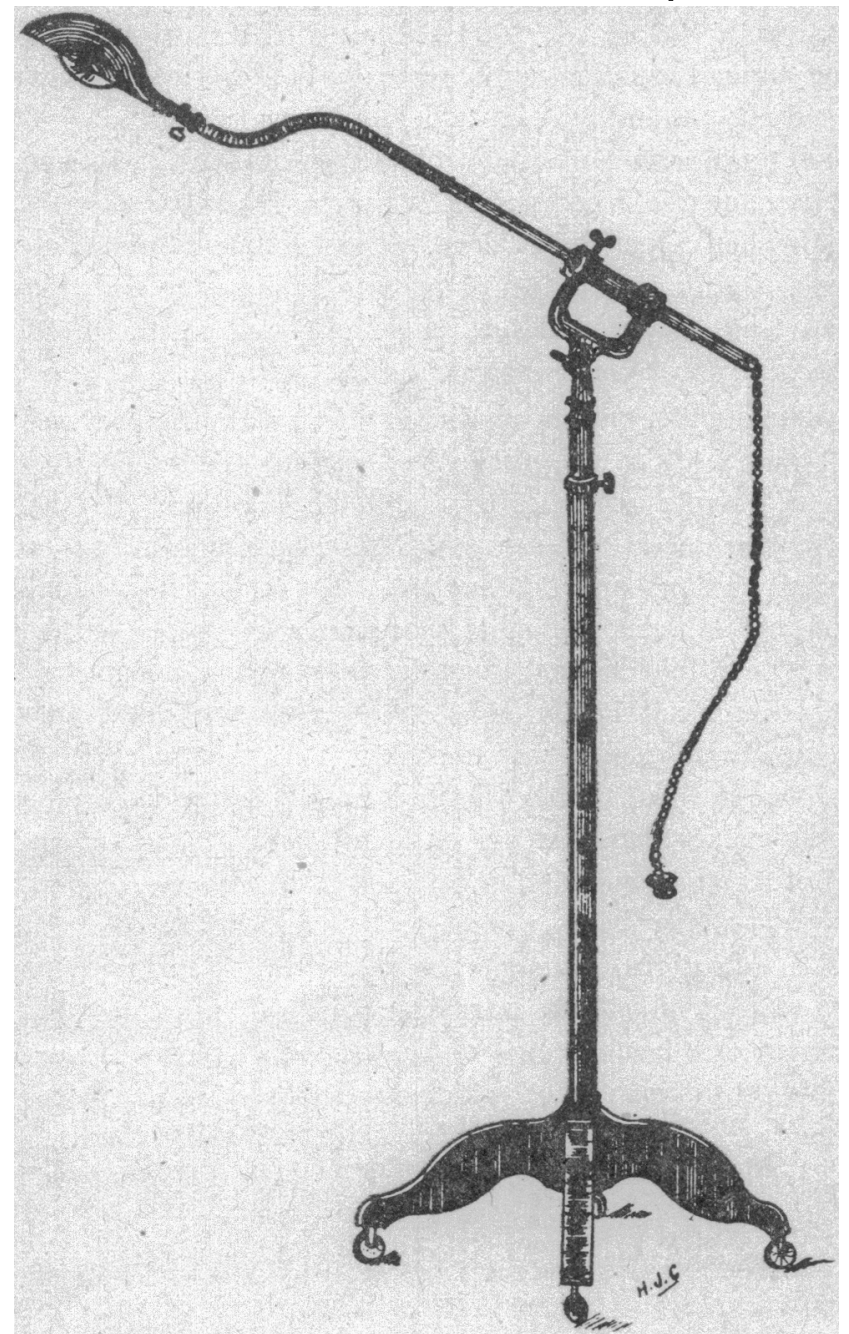

by a thumb screw. At the top of the inner tube a slot engages a U-shaped bracket, which can be tilted up or down and is retained in position by a winged screw. A short tube is brazed into the ends of the bracket.

The upright piece of an ordinary goose-neck electric light stand is pushed through the tube in the bracket, where it can be clamped if necessary. The flexible part of this horizontal arm is of sufficient length (eight inches) to enable one to turn the light in any desired direction.

The lamp itself is a spiral filament stereopticon of 24 candle-power with no frosting and is shaded by an aluminum reflector so fastened as to allow of its being turned about the lamp in directing the light toward any side.
This apparatus has been found especially useful in mastoid work, for which, indeed, it was originally intended. The stand is placed behind the anesthetist and the horizontal arm, covered with sterilized towels, is stretched forward over the patient, where the light, with the aid of one hand only, can be instantly and easily adjusted to illuminate the operative field or turned up toward the head-mirror of the operator. This appliance has been in use in the New York Eye and Ear Infirmary for the past year, and has given satisfaction. It is manufactured by the Hospital Supply Company of New York.

12 Carlton Street

\section{ACUTE FRONTAL SINUSITIS.}

C. M. HARRIS, M.D.

Ophthalmologist to the Conemaugh Valley Memorial Hospital. JOHNSTOWN, PA.

Although writers frequently refer to ocular phenomena occurring as complications or symptoms of disease of the accessory cavities of the nose, I report this case in order to emphasize the importance of differential diagnosis and to illustrate the fallacy of too strictly limiting one's observation to the organ which exhibits the more pronounced symptoms.

History.-A minister, aged 39 , gave a history of good general health except for a chronic rhinitis of moderate degree. He had worn glasses during the past sixteen years. The latest pair proved to be lenses for the correction of simple myopia. I later found a marked degree of compound myopic astig. matism, which being corrected, greatly improved the vision. The patient had always been singularly free from the usual symptoms of eyestrain, seeking glasses only as a means of improving vision. In the latter part of the winter of 1897 he contracted a "cold" which was followed by excruciating pain at the root of the nose on the right side,. extending outward along the supraorbital region and upward toward the vertex. The right eye was painful and sensitive to light. He consulted an ophthalmologist in the city where he then lived, who pronounced it "optic nerve inflammation," and proceeded to treat the eyes. After a week of almost unbearable pain, a discharge of greenish pus appeared in the right naris and relief was prompt. Almost four years later after moving to another state, a similar attack developed. In spite of the previous history, another specialist treated the eye without so much as examining the nasal cavities. Another week of suffering, a flow of pus, and it terminated as before. In February of this year I saw him for the first time. He related his history and stated that he had recently been suffering from a "cold," For two days past excruciating pain in the right inner supra-orbital and frontal regions and right eye had prevailed.

Examination.-Light pressure over the eye and frontal sinus could hardly be borne. The eye was also exceedingly sensitive to light. The vision on comparison with the fellow eye, and with test after recovery, was about what one would expect in neglected myopia with astigmatism. The fundus appearance can be described in the same way. The left nasal passage was negative except for a moderate rhinitis. The right side, however, presented more rhinitis, and the middle turbinate was turgid and had a glazed appearance.

Treatment.-After application of adrenalin and cocain an attempt was made to probe the frontal duct but it proved futile. An alkaline wash and an adrenalin spray were prescribed for nasal application. Acetanilid compound was given for pain, and atropin for its effect on the mucous membrane. He returned next day, reporting slight relief. Another attempt at probing proved successful. A small amount of pus appeared and relief was .almost immediate. The turgidity of the middle turbinate and the flow of pus continued for about ten days. Absolutely no subjective symptoms have since been present. 